\title{
LAS UNIVERSIDADES ESPAÑOLAS EN LOS RANKINGS INTERNACIONALES. LOGROS, CARENCIAS Y DESAFÍOS
}

\section{SPANISH UNIVERSITIES IN INTERNATIONAL RANKINGS. ACHIEVEMENTS, SHORTCOMINGS AND CHALLENGES}

http://dx.doi.org/10.15304/ie.28.5187

\author{
Tania Blanco Sánchez \\ Universidad de Cádiz \\ tania.blanco@uca.es
}

\section{RESUMEN}

Hoy en día existen un gran número de rankings internacionales de universidades, los cuales no se pueden ignorar porque de forma directa o indirecta están afectando a toda la comunidad universitaria española. El objetivo de este artículo se centra en ofrecer una visión crítica de la posición que ocupan las universidades españolas en los rankings internacionales analizando su contexto. Para ello, el presente trabajo realiza en primer lugar una la revisión de los cambios más recientes y de las características de las universidades españolas. Esto nos permiten visualizar el contexto en el que se encuentra el sistema universitario español. A continuación, se describen los rankings internacionales más importantes del mundo. En último lugar, se analizan e interpretan los datos aportados reflexionando sobre el porqué de esta situación. Dichas reflexiones son claves para que las universidades puedan obtener una mejor posición en los rankings internacionales en los años venideros.

Palabras clave: Educación Superior; Universidad; Ranking Internacional.

\begin{abstract}
Today there are a large number of international rankings of universities, which can not be ignored because directly or indirectly they are affecting the entire Spanish university community. The aim of this article is to offer a critical view of the position of Spanish universities in international rankings by analyzing their context. For this, the present work carries out in the first place a review of the most recent changes and the characteristics of Spanish universities. This allows us to visualize the context in which the Spanish university system is located. Next, the most important international rankings in the world are described. Lastly, the data provided is analyzed and interpreted, reflecting on the reason for this situation. These reflections are key for universities to obtain a better position in the international rankings in the years to come.
\end{abstract}

Keywords: Higher Education; University; International Ranking. 


\section{PRESENTACIÓN}

En la actualidad los rankings universitarios han adquirido una gran importancia e interés, tanto a nivel nacional como internacional. En este sentido, este artículo pretende aportar una visión general de por qué las universidades españolas no aparecen en los 100 primeros lugares en los dos principales rankings internacionales: Academic Ranking of World Universities y The Times Higher Education World University.

En este trabajo se analizan las características de las universidades españolas con el objetivo de identificar sus problemáticas y, en consecuencia, exponer algunas consideraciones respecto a la posición de las universidades españolas en los rankings internacionales. Asimismo, se realiza una amplia reflexión sobre las deficiencias existentes en el sistema universitario español.

Dos cuestiones fueron las que guiaron el presente trabajo: ¿Cuál es la posición de las universidades españolas en los rankings internacionales?, y ¿por qué las universidades españolas ocupan esas posiciones en las clasificaciones internacionales?

El objetivo de este artículo se centra en ofrecer una visión crítica de la posición que ocupan las universidades de España los rankings internacionales analizando su contexto (características y cambios en el sistema universitario español, la globalización e internacionalización de las universidades españolas, y los rankings internacionales). Además, se proponen una serie de recomendaciones para mejorar las posiciones de las universidades españolas en los rankings internacionales.

Hemos estructurado este artículo en tres apartados. El primero sirve para contextualizar las universidades españolas a partir de sus características y los cambios más recientes que han sufrido las universidades, en la que se aportan las cifras más importantes acerca de estas instituciones. En el segundo, se exponen los principales rankings internacionales y la situación al respecto de las universidades españolas. En último lugar, se ofrecen un análisis de los principales problemas que a nuestro parecer impiden que las universidades españolas consigan un mejor posicionamiento en las clasificaciones internacionales. Este análisis puede ser de gran utilidad para aquellas universidades españolas que pretendan alcanzar un posicionamiento más alto en los rankings internacionales.

\section{LAS CARACTERÍSTICAS DE LAS UNIVERSIDADES ESPAÑOLAS}

Las características actuales de las universidades españolas son fruto de la sociedad en la que se encuentran inmersas. Estas características han permitido a las universidades españolas redefinir sus funciones y resistir en el contexto contemporáneo, un tanto adverso. La reforma del sistema universitario, la globalización e internacionalización de las universidades y el Espacio Europeo de Educación Superior, son los principales retos a los que se han tenido que enfrentar estas instituciones de educación superior. 


\subsection{Los cambios en el sistema universitario español}

En los últimos años, la universidad española ha sufrido un proceso de reestructuración y adaptación como consecuencia de varios factores: la legislación y el entorno político, la demografía, la competencia y la tecnología.

\subsubsection{Legislación y entorno político}

Uno de los cambios más significativos en el sistema universitario español se produjo con la aprobación de la Ley de Reforma Universitaria en 1983. Esta ley supuso el inicio de una transformación en la Educación Superior, que propinó la descentralización de la educación universitaria y la transferencia de competencias en esta materia a las Comunidades Autónomas.

A la descentralización de las universidades hay que sumar otras transformaciones que se han producido en el seno de la universidad española.

\subsubsection{Demografia}

Otra modificación importante hace referencia a la demografía, y es que la universidad española ha dejado de ser una universidad formadora de elites para convertirse en una institución más abierta a la ciudadanía, y se ha multiplicado el número de alumnos, sobre todo el número de mujeres que supera al de hombres. Por todo ello, el perfil de la universidad española ha cambiado de manera significativa. Pero esta transformación es fruto de una evolución, en la que se diferencian tres etapas, tal y como muestra la tabla 1.

\begin{tabular}{|l|l|l|}
\hline Etapa & Período & Características \\
\hline Universidad Elitista & Hasta 1959 & $\begin{array}{l}\text { Normalmente sólo estudiaban los hombres de familias } \\
\text { adineradas. }\end{array}$ \\
\hline Universidad Masificada & Hasta 2000 & El porcentaje de mujeres es ya superior al de hombres. \\
\hline Universidad Universal & A partir de 2000 & $\begin{array}{l}\text { Se produce un afianzamiento de las nuevas tecnologías y } \\
\text { comienzan a romperse las fronteras espaciales. }\end{array}$ \\
\hline
\end{tabular}

Tabla 1. Etapas recientes de la universidad española. Fuente: Castillo (2007).

La última etapa se refuerza con la entrada en vigor de la Ley Orgánica de Universidades 4/2007, por la que se modifica la Ley 6/2001. Esta ley se enmarcaba dentro del proceso estratégico de mejora y modernización de las universidades españolas, con la adaptación al Sistema Universitario Español (SUE) al Espacio Europeo de Educación Superior (EEES) y la incorporación de la investigación académica al proyecto del Espacio Europeo de Investigación. Las medidas universitarias más importantes de esta ley que modificaban el sistema universitario eran:

- Estructurar las enseñanzas universitarias en tres ciclos: grado, máster y doctorado, cuya superación da lugar a los títulos oficiales correspondientes. 
- Adoptar medidas para facilitar la transferencia del conocimiento e investigación a la sociedad. Incluso se impulsaba la excedencia temporal de los investigadores al servicio de empresas de base tecnológica (impulsan las spin-off).

- Adoptar medidas para facilitar la efectividad del Espacio Europeo de Enseñanza Superior, mediante programas de movilidad de profesores y alumnos, así como armonización de títulos españoles y europeos.

Otro cambio importante en las universidades españolas fue la aprobación de la Ley Orgánica de Universidades, que entró en vigor en 2002. Esta ley supuso un aumento importante de las universidades privadas, ya que igualaba este tipo de universidades a las públicas. Así, en España en los últimos años el número de universidades privadas ha aumentado de manera significativa.

\subsubsection{Competencia}

Hasta el año 1952 había en España 4 universidades privadas todas ellas pertenecientes a la Iglesia (Deusto, Pontificia de Comillas, Pontificia de Salamanca y Navarra). Durante los siguientes 40 años no se constituyó ninguna universidad privada nueva hasta la creación en 1991 de la Universidad Ramón Llull, en Barcelona. Esta universidad se creó cuando el gobierno español aceptó que se constituyesen centros privados de educación superior que no tuviesen vinculación con la Iglesia. A partir de ese año se acelera la creación de universidades privadas, siendo en los últimos años cuando se ha producido un crecimiento sustancial. Actualmente en España existen 33 universidades privadas, de las que 11 pertenecen a la Iglesia y el resto son universidades corporativas, que se deben a iniciativas empresariales. Este incremento de universidades privadas ha endurecido la competencia universitaria. Tanto es así, que la Conferencia de Rectores de las Universidades Españolas (CRUE) señaló que las “universidades 'convencionales' se enfrentan a muchos retos, entre los que destaca la pérdida de la exclusividad en la formación superior, con la aparición de nuevos agentes formativos como son las 'universidades de empresa'. Estas 'universidades corporativas' poseen un enorme potencial de crecimiento" (2004: 27).

\subsubsection{Tecnología}

En los últimos tiempos, gracias a los avances tecnológicos se han creado otro tipo de universidad cuya docencia se imparte a distancia, son las denominadas universidades no presenciales. Estas universidades también son conocidas como universidades online o universidades virtuales. Actualmente, en el sistema universitario español existen seis universidades cuya docencia es no presencial.

\subsubsection{Cifras anuales}

En general, la universidad española es de grandes dimensiones, es decir que está compuesta por un número amplio de estudiantes. Tiene una media de 22.351 estudiantes, la segunda más alta en Europa después de Italia (23.537) y 3,5 veces superior a la media de la universidad de la Unión Europea (6.322 estudiantes) o Estados Unidos (8.490), (De Miguel, Vaquera y Sánchez, 2005). 
En el momento de la realización de este estudio (2016-2017), en el sistema universitario español existían 83 universidades en las que se impartían docencia oficial: 50 públicas (47 presenciales, 1 no presencial y 2 universidades especiales -UIMP y UNIA-) y 33 universidades privadas ( 28 presenciales y 5 no presenciales).

Respecto a los datos sobre las universidades españolas, en el curso 2016-2017 en el SUE se elevó ligeramente el número de matrículas en la universidad española respecto a los 3 últimos años hasta llegar 1.558.685 alumnos en los estudios de grado, primer y segundo ciclo, máster y doctorado.

En lo relativo a la docencia, en el sistema universitario español hay un total de 115.366 profesores, de los cuales 45.839 son funcionarios. De estos funcionarios más de un $23 \%$ son mayores de 60 años y casi un 10\% mayor de 65 años. De todos ellos, solo los catedráticos tienen dos o más sexenios 'vivos"1 de media, mientras que los profesores titulares apenas sobrepasan el 1,5 de sexenio y los catedráticos de escuelas universitarias apenas llegan al sexenio.

\subsection{La globalización e internacionalización de las universidades}

Uno de los fenómenos que han afectado a las universidades españolas es la globalización. Este fenómeno tiene tal importancia que algunos autores consideran que a las funciones clásicas de la universidad (la docencia, la investigación y la extensión) hay que añadir la internacionalización y la regionalización.

Según la UNESCO (1998), la globalización incorpora nuevos e importantes temas en el ámbito de la educación, la investigación, lo científico, pero también en el ámbito social y cultural. Por ello, en un mundo en el que cada vez se exigen mayores competencias, los centros de enseñanza superior deben ser unas plataformas internacionales vitales para la colaboración y el diálogo, así como para el intercambio y análisis de nuevas ideas innovadoras.

Algunas políticas supranacionales junto con la internacionalización de las profesiones y de la actividad académica, han contribuido de forma decisiva a la globalización de la educación superior. Esto ha conllevado a la convergencia de programas y modelos de enseñanza, y al aumento de la competencia entre las universidades (Íñiguez, 2009). Por ejemplo, con el llamado Proceso de Bolonia se adapta el contenido de los estudios universitarios a diversos países y se facilita el intercambio de titulados entre los países pertenecientes a ese proceso.

Por otra parte, la internacionalización implica una disminución de las barreras de entrada o de movilidad entre instituciones educativas abriendo sus campus y ofreciendo programas en los distintos países. Así, supone una mayor concentración de la oferta universitaria, con el desarrollo de grandes universidades y de centros especializados. Algunas de las estrategias de internacionalización más empleadas por las universidades desgranadas por las universidades en la Estrategia para la internacionalización de las Universidades Españolas 2015-2020 son: la apertura de campus, las

Es el tramo de investigación que suponen el complemento de productividad más importante en la universidad sus últimos años. 
fusiones y adquisiciones, las franquicias, la formación en línea y los programas blended (aprendizaje combinado de enseñanza presencial con la tecnología no presencial) y las alianzas estratégicas.

Con estas estrategias se consigue que la internacionalización poco a poco aumente, tal y como está sucediendo en las universidades de España. Cada año la cifra de estudiantes extranjeros que cursan en nuestro país es mayor, según los datos del Ministerio de Educación, Cultura y Deporte (2017). Así, en el curso 2015-16 el número total de estudiantes extranjeros universitarios en España fue de 85.973 alumnos, frente a los 56.630 del 2007-08. En cuanto al personal docente e investigador (PDI), solo un 2,4\% es extranjero, siendo apenas de un 2,0\% en las universidades públicas y un 4,9\% en las universidades privadas.

Una de las consecuencias de la globalización o internacionalización es la creación del Espacio Europeo de Educación Superior (EEES), conocido también como el Plan Bolonia. El EEES ha tenido un gran efecto en el sistema universitario español. Así, según el Ministerio de Educación Cultura y Deporte (2017), buena parte de los estudiantes extranjeros del curso 2015-16, en concreto 30.992 alumnos, procedían de los 27 países miembros de la Unión Europea. Algo similar ocurre con los profesores, ya que más de $65 \%$ de los profesores extranjeros vienen de la Unión Europea.

\section{LOS RANKINGS INTERNACIONALES COMO GUÍAS NECESARIAS}

En el nuevo mundo globalizado basado en la economía del conocimiento, las universidades desarrollan un papel fundamental como motor de un sistema de formación y de producción científica. La globalización y el EEES han aumentado significativamente la competitividad en las universidades. A lo que se suman los cambios acaecidos en los últimos años en el mundo universitario como el aumento de la demanda de la sociedad, la reducción de la financiación o la influencia de las empresas (Carrillo, Castillo y Blanco, 2015).

La competencia entre universidades se observa de manera visible en los rankings universitarios. Estos rankings "inevitables y probablemente necesarios" (Altbach, 2006: 80) clasifican a las mejores universidades según su calidad y prestigio. Las clasificaciones universitarias reflejan la batalla por controlar los flujos de conocimiento en el mundo actual y los rankings ayudan en el intento (Luque-Martínez, Faraoni y Doña-Toledo, 2018). Por un lado, las universidades que tradicionalmente han dominado la producción científica internacional quieren mantener su posición, por otras universidades emergentes quieren tener una presencia competitiva. Para establecer un listado de las mejores instituciones de educación superior se realizan los rankings, que las ordenan apareciendo en primer lugar aquella institución que ha obtenido una mayor puntuación en los indicadores de acuerdo a una metodología (Martínez Rizo, 2011).

Estas clasificaciones se pueden utilizar como herramienta comparativa por parte de las instituciones de educación superior. Además, se dilucida como un indicador de calidad universitaria o una aproximación a garantía de calidad que informa a sus públicos objetivos. Es decir, que las clasificaciones son una guía para ayudar a los estudiantes a elegir la universidad en la que desean formarse y desarrollarse, y a los empresarios en su búsqueda de graduados excelentes. Por lo que, además, las clasificaciones universitarias sirven para optimizar la calidad de sus sistemas y aumentar la satisfacción de su público. 
Por último, las instituciones de educación superior también utilizan las clasificaciones para fines comerciales y de publicidad, y para establecer estrategias que mejoren su enseñanza. Es decir, que los grupos de interés aprecian el reconocimiento de las universidades en los distintos rankings por el nacimiento de instrumentos que intentan sintetizar el puesto de las universidades (DelgadoLópez-Cózar, 2012).

\subsection{Historia de los rankings internacionales}

A pesar de que los rankings hayan aparecido por primera vez en 1870 (Grewal et al., 2008). El fenómeno de los rankings universitarios es relativamente reciente. La fama de los rankings de universidades adquiere una relevancia especial con la publicación del Academic Ranking of World Universities (ARWU). Fue en 2004 cuando se presentaron los dos primeros rankings internacionales: el The Times Higher Education World University Ranking y el Academic Rankings of World Universites (ARWU). Ambos rankings realizan su clasificación de manera independiente y con criterios distintos, pero coinciden en la identificación de las mejores universidades del mundo. Las diez mejores universidades eran y siguen siendo prácticamente las mismas, predominando las universidades norteamericanas y las británicas.

El análisis de las universidades se fundamenta en la compilación de datos de las instituciones universitarias a partir de estadísticas oficiales o de la información aportada por las propias universidades, y de las encuestas de opinión.

Tal y como señalan Gormley y Weimer (1999), dicha clasificación se elabora con una metodología particular, y en la elaboración de los criterios intervienen numerosos factores, simplificados en una clasificación numérica.

Actualmente existen diversos: rankings nacionales en más de cuarenta países y rankings internacionales que comparan y clasifican universidades a escala global. A continuación, se muestra la tabla 2 que recopila las clasificaciones universitarias internacionales y nacionales más importantes.

Dentro de las diversas clasificaciones existentes que evalúan a las universidades internacionales, se han escogido aquellas clasificaciones que se ajustan a una serie de criterios que se consideran necesarios para posteriormente analizar posición de las universidades españolas. Los criterios empleados en la selección de estas clasificaciones internacionales son los siguientes:

1. Satisface los principios de Berlín (UNESCO, 2006).

2. Es relevante o tiene cierto prestigio en el ámbito de la educación universitaria.

3. Su metodología está basada en criterios relativamente objetivos.

4. Analiza a las universidades del mundo, por lo que su clasificación tiene internacional o global.

5. Estudia a las institucionales, es decir, que abarca a toda la unidad educativa de manera global, no determinados aspecto o escuelas. 


\begin{tabular}{|c|c|c|}
\hline Internacional & Nacional & País \\
\hline \multirow{6}{*}{$\begin{array}{l}\text { The Guardian } \\
\text { The Philosophical } \\
\text { Gourmet } \\
\text { Qué Pasa } \\
\text { Webometrics Rankings of World } \\
\text { Universities } \\
\text { CEST-suiza } \\
\text { The Economist } \\
\text { The Financial Times } \\
\text { Universidad Jiao Tong de Shangai } \\
\text { Times Higher Education } \\
\text { Supplement } \\
\text { Scimago } \\
\text { Webometrics } \\
\text { U-MULTIRANK } \\
\text { Round University Ranking } \\
\text { Center for World University } \\
\text { Ranking } \\
\text { Leiden Ranking } \\
\text { US News \& World Report Ranking }\end{array}$} & $\begin{array}{l}\text { Diario El Mundo } \\
\text { Ranking universidades españolas } \\
\text { (Gaceta Universitaria) } \\
\text { COTEC } \\
\text { Ranking de productividad en investigación de } \\
\text { las universidades públicas españolas } \\
\text { (Universidad de Granada) }\end{array}$ & España \\
\hline & $\begin{array}{l}\text { The Sunday Times } \\
\text { The Guardian University Guide } \\
\text { NUBS (transferencia) }\end{array}$ & Reino Unido \\
\hline & $\begin{array}{l}\text { Melbourne Institute } \\
\text { The Good University Guide } \\
\text { Hobsons }\end{array}$ & Australia \\
\hline & $\begin{array}{l}\text { CHE-Die Zeit } \\
\text { B-School } \\
\text { Center of Excellence } \\
\text { Women and Science }\end{array}$ & Alemania \\
\hline & $\begin{array}{l}\text { Le Nouvel Observateur } \\
\text { Le Point }\end{array}$ & Francia \\
\hline & $\begin{array}{l}\text { Business Week } \\
\text { Gourman } \\
\text { The Center (Investigación) } \\
\text { The Wall Street Journal } \\
\text { NewsWeek Internacional } \\
\text { AUTM (transferencia) } \\
\text { Journal of Blacks in Higher Education* } \\
\text { (compromiso social) } \\
\text { New Mobility Magazine } \\
\text { (compromiso social) }\end{array}$ & Estados Unidos \\
\hline
\end{tabular}

Tabla 2. Rankings universitarios en función del ámbito en el actúan. Fuente: Elaboración propia a partir de Fundación CYD (2008).

Así, de todas las clasificaciones expuestas en la tabla 2 se han seleccionado las clasificaciones de Academic Ranking of World Universities y The Times Higher Education World University, ya que eran los dos rankings que más se ajustaban a las especificaciones comentadas. Esta selección se ve reforzada por otros argumentos como ser los dos rankings internacionales pioneros o ser las dos clasificaciones universitarias más importantes del mundo (Euobserver, 2010).

\subsection{Academic Ranking of World Universities}

El propósito inicial de la Academic Ranking of World Universities (ARWU) era medir la posición global de las principales universidades de China, pero con el tiempo se ha convertido en la clasificación de referencia para medir la posición de las universidades analizadas respecto a 
la comunidad universitaria global. El Ranking Académico de Universidades Mundiales (ARWU) publicado por investigadores de la Universidad Jiao Tong de Shanghai se ha convertido en una importante fuente de información. El ranking de Shanghai es un recurso importante para explorar las características y calidad de las instituciones académicas y sistemas universitarios a nivel mundial. La accesibilidad de las fuentes de los datos en bruto y el hecho de que la jerarquía de universidades se alinea aproximadamente con las percepciones del desempeño histórico y reciente de las universidades de investigación de élite han contribuido a su aceptación y éxito (Docampo y Cram, 2014).

La ARWU realiza una primera selección a partir de aquellas universidades que tienen un premio Nobel, un ganador de una medalla Fields, un investigador muy citado o publicaciones en Nature o Science. No obstante, también incluye a las mayores universidades de cada país que tienen un número significante de artículos indexado en Science Citation Index-Expanded (SCIE) y Social Science Citation Index (SSCI). Por lo que clasifica a más 800 universidades anualmente, destacando especialmente las 500 mejores en su web.

Las universidades son clasificadas a partir de la puntuación obtenida en seis indicadores: estudiantes o antiguos estudiantes, y profesores ganadores de los Premios Nobel y Medallas Fields, los investigadores altamente citados, artículos publicados en Nature y Science, los documentos indexados en los principales índices de citas (SCIE y SSCI), y el per cápita de rendimiento académico de una institución.

Las calificaciones de cada indicador se ponderan para obtener una puntuación final global de una institución. Para cada indicador, la institución con mejor puntuación se le asigna una puntuación de 100, y el resto de universidades se calculan como un porcentaje de la puntuación máxima. Los porcentajes que se asignan son los siguientes:

- Número de alumnos que han ganado el Premio Nobel y Medallas Académicas (10\%).

- Número de miembros de la plantilla del cuerpo docente e investigador que han ganado el Premio Nobel y Medallas Académicas (20\%).

- Número de investigadores más citados dentro de su campo de especialización (20\%).

- Número de artículos publicados en las revistas Science y Nature (20\%).

- Calidad de los artículos (20\%).

- Rendimiento académico per cápita de una institución (10\%).

La metodología de la ARWU ha sido criticada por favorecer a las universidades anglosajonas. Primero por el sesgo idiomático de las publicaciones, que son casi exclusivas de habla inglesa, y segundo por la supremacía de la investigación, con la selección de los premios y la experiencia de los ganadores de los premios, que no se otorgan por calidad, sino por ser el resultado de proceso político.

No obstante, esta clasificación se basa en un enfoque objetivo con una metodología transparente y consistente (datos bibliométricos y otros indicadores). El rector de la Universidad 
de Oxford, Chris Patten (2003), ha señalado que la metodología es bastante sólida realizando una justa clasificación. Margison (2007) también ha comentado que la clasificación ARWU tiene un fuerte rigor académico. Además, Bollag (2006) señala que la credibilidad de esta clasificación mundial es muy alta, por lo que el análisis de sus resultados es importante e influyentes a nivel internacional.

En este sentido, el impacto del Ranking (ARWU) a nivel mundial ha sido considerable. Tanto es así que se le considera uno de los responsables de los cambios en políticas de educación superior de países como Francia o Rusia. Enserink (2007) explicó que la pobre actuación de Francia en el ranking de Shanghai ayudó a generar un debate nacional sobre la educación superior que dio lugar a una nueva ley dando a las universidades más libertad.

En 2018 el ranking de Shanghái vuelve a situar en las mejores posiciones a universidades estadounidenses. El primer puesto es para Harvard y la segunda posición es para Stanford. Estas dos universidades mantienen los puestos de ediciones anteriores. El tercer lugar recae en Reino Unido, en la Universidad de Cambridge. Le siguen el Instituto de Tecnología de Massachusetts (MIT), y las universidades de California, Princeton, Oxford y Columbia. Cierran el top ten el Instituto de Tecnología de California y la Universidad de Chicago. Estas diez primeras posiciones en el ranking son las mismas que las del año 2017.

\subsection{The Times Higher Education World University Ranking (THES)}

The Times Higher Education World University Rankings es la clasificación que realiza Times Higher Education Supplement (THES-Ranking) sobre más de 1.000 universidades en el mundo. Se trata del único ranking mundial elaborado por una consultora independiente PricewaterhouseCoopers (PwC).

Esta clasificación analiza las principales funciones de las universidades: enseñanza, investigación, transferencia de conocimientos y perspectivas internacionales. Para ello, utilizan 13 indicadores calibrados a los que se les asignan los siguientes porcentajes:

1. Enseñanza (el entorno de aprendizaje): 30\%

- Reputación: $15 \%$

- Proporción de personal a estudiante: $4,5 \%$

- Relación doctorado-graduado: 2,25\%

- Relación entre doctorado y académico: $6 \%$

- Ingresos institucionales: $2,25 \%$

2. Investigación (volumen, ingresos y reputación): 30\%

- Encuesta de Reputación: 18\%

- Ingresos de investigación: 6\%

- Productividad de la investigación: 6\% 
3. Citas (influencia de la investigación): 30\%

4. Perspectivas internacionales (personal, estudiantes, investigación): 7,5\%

- Relación internacional-estudiante-estudiante: $2,5 \%$

- Relación entre el personal internacional y el personal doméstico: 2,5\%

- Colaboración internacional: $2,5 \%$

5. Ingresos de la transferencia de conocimiento: $2,5 \%$

El centro de la metodología se basa en la realización de dos encuestas y en el análisis de datos estadísticos en cinco ámbitos: enseñanza, investigación, citas, perspectivas internacionales e ingresos por transferencia de conocimientos.

\section{Encuestas}

Respecto a las encuestas, se realizan a más de 10.000 personas del sector académico. Las respuestas son estadísticamente representativas en la composición geográfica y del ámbito académico mundial. Los datos de 2017 se combinan con los resultados de la encuesta de 2016, dando más de 20.000 respuestas.

El indicador más destacado en la investigación es la encuesta de reputación, que se centra en la reputación de excelencia en la investigación. Esta encuesta se basa en las respuestas de la encuesta anual de la reputación académica.

\section{Datos estadísticos}

Respecto a los ingresos institucionales y de investigación se comparan los datos del personal académico y se normaliza para obtener la paridad de poder adquisitivo (PPP) en los distintos países. Este indicador muestra la situación económica general de una institución, así como de la infraestructura y de las instalaciones disponibles para los estudiantes y el personal.

Para medir la productividad se basan en el número de trabajos publicados en las revistas académicas indexadas por la base de datos Scopus de Elsevier. Esto proporciona datos sobre la producción de las universidades que publican artículos en revistas de calidad revisadas por pares.

En cuanto al indicador de influencia en la investigación (citación), se examina el papel de las universidades en la difusión de nuevos conocimientos e ideas. Para ello, examinan la influencia de la investigación detectando el número de veces que el trabajo publicado por una universidad es citado por los académicos en todo el mundo, utilizando la base de datos del presente año de los datos bibliométricos de Elsevier.

El tercer indicador denominado perspectivas internacionales, se calcula en proporción a las publicaciones de investigación de la universidad que tienen al menos un coautor internacional.

Por último, este ranking analiza la capacidad de una universidad para ayudar a la industria con innovaciones, invenciones y consultoría. Esta categoría busca captar esta actividad de transferencia 
de conocimiento, analizando la cantidad de ingresos de investigación que una institución obtiene de la industria (ajustada por PPP), cotejado con la cantidad de personal académico que emplea.

En comparación con otros rankings (como la ARWU) aparecen bastantes menos universidades estadounidenses y más británicas. Las encuestas sobre el prestigio académico tienen el riesgo de favorecer a las universidades más conocidas, además, presupone que los académicos conocen realmente la calidad de investigación en su disciplina. Al igual que la ARWU, el índex de citas tiene sesgo idiomático que favorece al inglés.

Este ranking también ha recibido críticas por la volatilidad de los puestos que de algunas universidades en clasificaciones anuales de años diferentes. El THES está demasiado expuesto a la manipulación, ya que al cambiar los destinatarios de las encuestas o la forma en que los resultados del estudio se obtienen, el resultado puede ser cambiado notablemente. No obstante, realiza una evaluación a partir de diversas dimensiones como: investigación, enseñanza, empleabilidad y prestigio; y emplea en su metodología la combinación de dos técnicas distintas: el cuestionario y la recopilación de datos estadísticos.

El ranking The Times Higher Education World University de 2018 está liderado por las universidades inglesas de Oxford y Cambridge respectivamente, seguidas de las estadounidenses Instituto de Tecnología de California, Stanford e Instituto de Tecnología de Massachusetts. En sexta posición se sitúa, Harvard, seguida de Priceton, Imperial College Londo, ETH-Zurich, Universidad de Chicago y Universidad de California, en este orden. Así, la universidad de Cambridge ha pasado de ocupar la cuarta posición en 2017 al segundo lugar en el ranking de 2018, el resto de los diez primeros puestos no han sufrido cambios significativos. En general, las instituciones europeas ocupan la mitad de las 200 mejores universidades del mundo, con los Países Bajos y Alemania uniéndose al Reino Unido como los países más representados. Otra tendencia notable es el aumento de China. El gigante asiático ocupa dos posiciones en el top 30: Pekín y Tsinghua. En total, 77 países figuran en la clasificación.

El ranking de ARWU y el THES coinciden en las universidades que ocupan los primeros lugares, y en ambas clasificaciones se muestra una abrumadora supremacía de las universidades anglosajonas.

En estos dos rankings, ARWU y THES, las universidades españolas no ocupan buenas posiciones. En la clasificación de The Times Higher Education World University Ranking 2018, solo hay tres universidades españolas entre las 250 mejores del mundo. La Pompeu Fabra en la posición 140, la Autónoma de Barcelona en el lugar 147, la y la Universidad de Barcelona más allá del puesto 200. En total, 7 universidades entre las 500 primeras del mundo.

Mientras que en el ARWU 2018, la primera universidad española, que está en el puesto 179, es la Universidad de Barcelona, seguida de la Complutense de Madrid, en el 212, y la Universidad Pompeu Fabra, en el lugar 239. En total, tan solo 10 instituciones educativas españolas se cuelan en los 500 primeros puestos. Lejos quedan Estados Unidos (139) China (56), Reino Unido (39), Alemania (36) y Australia (23). 


\section{LA PROBLEMÁTICA DE LAS UNIVERSIDADES ESPAÑOLAS EN LOS RANKINGS INTERNACIONALES}

Tras analizar de forma exhaustiva el estado de la cuestión a través de la bibliografía existente sobre la temática, los informes del Ministerio de Educación de España y los dos principales rankings internacionales universitarios, se puede afirmar que España está entre los veinte países más avanzados económicamente del mundo, pero no aparece en la élite universitaria internacional. En este sentido, no se entiende que solo tenga una universidad entre las 200 mejores de todo el mundo en el ranking de Shanghái y dos universidades entre las 150 primeras del mundo en el ranking The Times; los dos rankings más importantes a nivel internacional. Es más, nunca una universidad española ha logrado posicionarse entre las 100 mejores del mundo en ninguno de estos dos rankings.

A todo ello hay que sumar que las posiciones de las universidades españolas han ido disminuyendo en los últimos años, así si en el 2015 había trece universidades españolas entre las 500 mejores del mundo en el ranking ARWU, en 2016 bajaron a doce y en 2017 y 2018 se han reducido a once. Además, las mejores universidades españolas aparecen pasada la mitad de la tabla. En 2015 había 5 entre las mejores 250 universidades del mundo, en 2016 son 4, en 2017 son 3 y en 2018 se ha mantenido esa cifra de 3 . No obstante y a pesar de estos datos, puede atisbarse una ligera mejoría respecto al 2017. Así, las primeras universidades españolas en el año 2017 del ranking The Times ocupaban los puestos: 163, 175 por 140 y 147 en 2018; en el ranking ARWU ocurre algo similar: 239,261 y 268 por 179,212 y 239.

Uno de los elementos que ha dificultado la obtención de una mejor posición por parte de las universidades españolas ha sido las continuas modificaciones de las Leyes Orgánicas de Educación, que no ha reforzado la enseñanza superior de calidad. A todo ello hay que añadir la descentralización en materia de Educación Superior que ha permitido que las Comunidades Autónomas concedieran licencias de apertura a Universidades en todas las Comunidades, e incluso a buena parte las provincias y ciudades medias. Esto ha provocado la dispersión de recursos, un exceso de oferta y dificultades para responder a estándares de excelencia. En España existen más de 80 universidades y 235 campus universitarios, mientras que por ejemplo en el estado norteamericano de California con la misma población que España existen apenas una decena de universidades, con varias de ellas presentes entre las mejores del mundo. Lo mismo ocurre con la oferta tan heterogénea y numerosa de titulaciones universitarias: más de 2.400 grados, 2.750 másteres y 1.680 programas de doctorado. Unas de cada tres de estas carreras universitarias apenas tienen 50 alumnos cada año. Eso ha conllevado a que las exigencias académicas han bajado notablemente y que las universidades privadas se hayan convertido en una "fábrica" de expedir títulos a cambio de dinero. En este sentido, Banacloche (2017) señala que el problema clave está en el control de calidad de los títulos y las universidades: no hay una forma homogénea de verificar los conocimientos con los que salen los estudiantes de cada centro. Pero no solo la universidad privada tiene problemas de control de los títulos. El Tribunal de Cuentas, en un informe sobre Universidades Públicas de 2015, solicitó simplificar la estructura de la Universidad, profesionalizar la toma de decisiones, "cambios en el proceso electivo que evite corporativismos", más transparencia y rendición de cuentas. 
Otro de los problemas de las universidades española es la cantidad y la calidad del profesorado. En 2015, había un profesor universitario por cada 13 estudiantes; frente a la media europea de 15 alumnos por docente. La ratio entre profesor y doctorandos en España es de tres a uno o superior, mientras que en las primeras universidades de los rankings es como mínimo de uno a tres. Es decir, que hay demasiados profesores permanentes y demasiados pocos doctorandos. De este modo, parecen existir una masa de profesores con plaza laboral fija que se han acomodado, y que no tiene un 'sexenio vivo'. Este aspecto perjudica enormemente a las universidades españolas debido a que los rankings miden, entre otras cosas, el número de publicaciones que los profesionales de las universidades realizan en revistas científicas de prestigio y el número de citas a los trabajos de sus investigadores. Por otro lado, existe otra masa de profesores universitarios en una situación precaria que carecen de puesto de trabajo fijo, encadenando contratos temporales, bajos salarios y sin disponer de unas condiciones laborales suficientes para poder investigar o producir.

No obstante, estos profesores son prolíferos generando más de 850.000 documentos generados al año, pero la relevancia de los artículos no es de gran calidad. Además, ese conocimiento no se transfiere a la economía real. España apenas genera el 0,8\% de las patentes mundiales, estando en las últimas posiciones de Europa y de los principales países desarrollados.

Además, el sistema de acceso del profesorado español es poco competitivo para atraer y conservar a los mejores docentes. Tanto es así, que siete de cada 10 profesores trabajan en la universidad en la que leyeron su tesis (Fundación CYD, 2015) siendo considerable la tasa de la llamada endogamia universitaria. Los docentes universitarios deberían ser las más importantes personalidades de cada materia, habiendo forjado su conocimiento en la experiencia profesional, lo que garantizaría una enseñanza exigente y de calidad. En este sentido, los años de experiencia tienen una influencia directa en las características que tienen los procedimientos implicados en la realización de las actividades que diseñan los profesores universitarios (De-Juanas y Ezquerra, 2014). Además, estos profesionales estarían en disposición de recibir un Premio Nobel o medalla de prestigio. Pero esto último es difícil de subsanar, ya que en el actual sistema educativo español los recursos económicos disponibles lo dificultan.

En cuanto a la internacionalización, a pesar de que España es el mayor importador de estudiantes de beca Erasmus, las universidades españolas es una de las que menos extranjeros incorporan en sus grados, másteres y doctorados. Pero esta falta de internacionalización es aún más patente en los profesores que apenas llegan al $2 \%$. A todo ello hay que sumar la diferencia entre el sistema educativo español y el del resto de sistemas europeos, con programas de difícil homologación en muchos casos y distintas aplicaciones y salidas profesionales.

Pero el principal problema de la universidad españolas es la reducción de la financiación de las universidades. En los últimos años, con la crisis y las políticas de austeridad, los recursos de las universidades han tendido a disminuido de manera significativa. Los rankings internacionales están copados por universidades anglosajonas, la mayoría de ellas privadas, que cuentan con un volumen desorbitado de recursos en comparación con las universidades españolas. Así las universidades de élite norteamericanas (Harvard, Princeton, MIT) tienen presupuestos de aproximadamente 150.000 euros por estudiante y año. Le siguen las universidades europeas que más invierten, Oxford y Cambridge 
con 50.000 euros por estudiante y año. La universidad española destina de promedio alrededor de 6.500 euros por estudiante y año. Los grandes presupuestos de que disponen las universidades de élite les permiten realizar grandes inversiones en investigación, lo que se traduce en: publicaciones que contabilizan como puntuaciones en componentes centrales del ranking, cautivar a los mejores docentes e investigadores y atraer a estudiantes de todo el mundo que están en condiciones de sufragar sus costosas matrículas o patrocinadores que lo hagan mediante becas de excelencia.

Por eso y para que las universidades españolas puedan competir con las mejores, tanto en Europa como en el resto del mundo es necesario que se hagan reformas profundas en su sistema de gobierno y de financiación, para que puedan verdaderamente competir con las mejores, tanto en Europa como en el resto del mundo (Galván, 2010).

A pesar del intento por parte del gobierno español en 2009 de mejorar la calidad del sistema universitario con el programa Campus de Excelencia Internacional para la agregación, especialización, diferenciación e internacionalización de sus mejores universidades, dicho programa terminó fracasando. Los motivos de dicho fracaso han sido varios: adolecer de una continuidad en su desarrollo; dificultades en la financiación de las Universidades, ligadas a las políticas de austeridad de gasto público de las Comunidades Autónomas; disminución en las líneas de subvenciones mediante proyectos del Plan Nacional de I+D + I y escasa capacidad de contratación con el sector productivo mediante actividades de transferencia y consultoría; ausencia de mecenazgo de cara a las universidades; y regulación rígida del sistema de educación superior, (Marcellán, 2013).

Por todo lo expuesto en este trabajo, se realizan una serie de recomendaciones concretas para la gestión y mejora de la posición de las universidades españolas en los rankings internacionales. Respecto a la Ley de Universidades, se deberían de establecer cambios a medio y largo plazo que guíen el desarrollo de las universidades españolas. Entre esos cambios, destacarían la centralización en materia de Educación Superior respecto a la cantidad y calidad de titulaciones universitarias. En lo relativo a la cantidad, las titulaciones deberían estar enfocadas siguiendo las tendencias del mercado laboral actual y las necesidades de la sociedad. En cuanto a la calidad, la ANECA debería de endurecer sus criterios y baremos a la hora de aprobar planes de estudios, así como limitar el poder de las Agencias de las Comunidades Autónomas (AAC, ACSUCYL, etc.) para asegurarse un estándar de calidad mínimo en todas las universidades españolas.

En lo relativo al personal docente e investigador, sería interesa crear un programa nacional para la contratación de profesores con reconocida trayectoria profesional, así como la internacional de profesores e investigadores universitarios. De este modo, se facilitaría la incorporación de profesorado de excelencia a la plantilla de las universidades. Por otro lado, para atraer al profesorado joven con talento sería necesario que estos docentes gozaran de promoción y seguridad laboral. En este sentido, se seguiría el ejemplo que aplican la mayoría de las universidades de Estados Unidos y Canadá, por el que un profesor ayudante o un profesor asociado promocionan hasta ser profesor titular. Asimismo, deberían eliminarse la acreditación para la contratación inicial y pasado un determinado periodo se les exijan requisitos de calidad en investigación. Además, sería importante promocionar la internacionalización de los docentes a través de programas con interés en $\mathrm{I}+\mathrm{D}+\mathrm{i}$. 
Por último, en el aspecto de financiación, el principal problema de las universidades españolas, se debería financiar a las universidades a partir de objetivos y resultados establecidos en una serie de programas, de este modo, se ligaría la financiación a los resultados y se incentivaría la calidad del profesorado. Igualmente habría que asignar un porcentaje de la financiación pública a las universidades, independientemente de su condición de pública o privada, a través de programas nacionales y autonómicos fundamentándose en la valoración de su actividad en función de resultados.

\section{REFERENCIAS BIBLIOGRÁFICAS}

Altbach, P. (2006). International higher education. Reflections on policy and practice. Boston: Editorial Boston College CIHE.

Banacloche, J. (5 de mayo de 2017). Madrid, la comunidad que tiene más universidades en España. Diario $A B C$, pp. 38-39.

Billaut, J. C., Bouyssou, D. y Vincke, P. (2009). Should you believe in the Shanghai ranking? Scientometrics, 84(1), 237-263. DOI: https://doi.org/10.1007/s11192-009-0115-X

Bollag, B. (2006). Group Endorses Principles for Ranking Universities. The Chronicle of Higher Education. Disponible en: http://chronicle.com/article/Group-Endorses-Principles-for/25703

Carrillo, V., Castillo, A. y Blanco, T. (2015). La proyección internacional de las universidades españolas en sus sedes webs. Opción, 31(1), pp. 183-203.

Castillo, A. (2007). La comunicación de las marcas universitarias españolas a través de sus sedes webs institucionales. Tesis Doctoral. Universidad de Extremadura.

CRUE (2004). Las Tecnologías de la Información y las Comunicaciones en el sistema universitario. Disponible en: http://www.crue.org (consultado el 18 de mayo de 2018).

CRUE (2015). La Universidad española en cifras. Disponible en http:/www.crue.org/SitePages/ La-Universidad-Espa\%C3\%B1ola-en-Cifras.aspx (consultado 5 de mayo de 2017)

De Miguel, J. M., Vaquera, E. y Sánchez, J. D. (2005). Spanish Universities and the Ranking 2005 initiative. Higher Education in Europe, 30(2), pp. 199-213. DOI: https://doi. org/10.1080/03797720500260256

De-Juanas, Á. y Ezquerra, Á. (2014). Experiencia profesional de los docentes universitarios que forman al profesorado de educación primaria y secundaria y sus tendencias metodológicas. International Journal of Developmental and Educational Psychology, 7(1), pp. 193-201.

Delgado-López-Cózar, E. (2012). Cómo se cocinan los rankings universitarios. How to cook the university rankings. Dendra médica, 11(1), pp. 43-58.

Docampo, D. y Cram, L. (2014). On the Internal Dynamics of the Shanghai ranking. Scientometrics, 98(2), 1347-1366. DOI: https://doi.org/10.1007/s11192-013-1143-0

Enserink, M. (2007). Who ranks the university rankers? Science, 317(5841), pp. 26-28. DOI: https:// doi.org/10.1126/science.317.5841.1026

Euobserver (2010). EU to test new university ranking in 2010. Disponible en https://euobserver. com/education/29189 (consultado el 15 de mayo de 2018).

Flórez-Parra, J. M.; López, M. V. y López, A. M. (2014). El gobierno corporativo de las universidades: Estudio de las cien primeras universidades del ranking de Shanghái Corporate gover- 
nance, analysis of the top 100 universities in the Shanghai ranking. Revista de educación, 364, pp. 170-196.

Fundación CYD (2008). Informe CYD 2008: La contribución de las universidades españolas al desarrollo. Barcelona: CYD.

Fundación CYD (2015). Informe CYD 2015: El papel de las universidades en España. Barcelona: CYD.

Galván, F. (2010). Las universidades españolas y los rankings internacionales. Diario ABC. Disponible en: http://www.abc.es/20101026/latercera/universidades-espanolas-rankings-internacionales-20101026.html

Gormley, W. y Weimer, D. (1999). Organizational Report Cards. Harvard: University Press, Massachusetts.

Grewal, R., Dearden, J. y Lilien, G. (2008). The University Rankings Game: Modeling the Competition among Universities for Ranking. The American Statistician, 62(3), pp. 232-237. DOI: https://doi.org/10.1198/000313008X332124

Íñiguez, S. (2009). La educación superior en un entorno global: estrategias de internacionalización de las universidades. La cuestión universitaria, 5, pp. 192-200.

Luque Martínez, T. Faraoni, N. y Doña-Toledo, L. (2018). Meta-ranking de universidades. Posicionamiento de las universidades españolas. Revista española de documentación científica, 41(1), p. 6. DOI: https://doi.org/10.3989/redc.2018.1.1456

Marcellán, F. (2013). La estrategia Universidad 2015: ¿Marcando un rumbo? Cuaderno de trabajo 1. Madrid: Studia XXI, Fundación Europea Sociedad y Educación. Recuperado de: http:// www.studiaxxi.com/site/wp-content/uploads/Cuaderno-Trabajo-1.pdf

Marginson, S. (2007). Global University Rankings: Implications in general and for Australia. Journal of Higher Education Policy and Management, 29(2), pp.131-142. DOI: https://doi. org/10.1080/13600800701351660

Martínez Rizo, F. (2011). Los rankings de universidades: una visión crítica. Revista de la Educación Superior, 40(157), pp. 77-97.

Ministerio de Educación, Cultura y Deporte (2014). Datos y cifras del sistema universitario español. Curso 2013-2014. Disponible en: https://www.mecd.gob.es/dms/mecd/servicios-al-ciudadano-mecd/estadisticas/educacion/universitaria/datos-cifras/datos-y-cifras-SUE-2013-2014. pdf

Ministerio de Educación, Cultura y Deporte (2015). Datos y cifras del sistema universitario español. Curso 2014-2015. Disponible en: https://www.mecd.gob.es/dms/mecd/servicios-alciudadano-mecd/estadisticas/educacion/universitaria/datos-cifras/Datos-y-Cifras-del-SUECurso-2014-2015.pdf

Ministerio de Educación, Cultura y Deporte (2016). Datos y cifras del sistema universitario español. Curso 2015-2016. Disponible en: https://www.mecd.gob.es/dms/mecd/serviciosal-ciudadano-mecd/estadisticas/educacion/universitaria/datos-cifras/datos-y-cifras-SUE2015-16-web-.pdf

Ministerio de Educación, Cultura y Deporte (2017). Estadísticas e Informes universitarios. Disponible en: https://www.mecd.gob.es/servicios-al-ciudadano-mecd/estadisticas/educacion/ universitaria/estadisticas.html 
Patten, C. (2003). Los méritos de Harvard para liderar el ránking de Jiao Tong. MBA \& Educación Ejecutiva. Disponible en: https://mba.americaeconomia.com/articulos/reportajes/los-meritos-de-harvard-para-liderar-el-ranking-de-jiao-tong

Portal de Gaceta Universitaria (2002). El sistema universitario español. Ranking de Universidades. Gaceta Universitaria. Disponible en: http://www.tugueb.com/e_campus/2002/06/reportaje/ ranking/3.html

Shanghai Ranking Consultancy (2018). Academic Ranking of World Universities 2018. Disponible en: http://www.shanghairanking.com/ARWU2018.html

Times Higher Education (2018). Times Higher Education World University Rankings 2018. Disponible en: https://www.timeshighereducation.com/world-university-rankings

UNESCO (1998). Declaración de la UNESCO sobre la Educación Superior en el siglo XXI. Disponible en: http://www.crue.org/dfunesco.htm

UNESCO (2006). Berlin Principles on Ranking of Higher Education Institutions. Disponible en http://www.che.de/downloads/Berlin_Principles_IREG_534.pdf 